OPEN ACCESS

Edited by:

Seter Siziya

Copperbelt University, Zambia

Reviewed by:

Ameer Ahmad,

Quaid-i-Azam Medical College,

Bahawalpur, Pakistan

E. Lisako J. McKyer,

School of Public Health, Texas A\&M University College Station,

United States

*Correspondence:

Takeo Fujiwara

fujiwara.hlth@tmd.ac.jp

Specialty section:

This article was submitted to

Child Health and Human

Development,

a section of the journal

Frontiers in Pediatrics

Received: 07 December 2017

Accepted: 20 April 2018

Published: 17 May 2018

Citation:

Tani Y, Fujiwara T, Ochi M, Isumi A and Kato $T$ (2018) Does Eating Vegetables

at Start of Meal Prevent Childhood

Overweight in Japan? A-CHILD Study.

Front. Pediatr. 6:134

doi: 10.3389/fped.2018.00134

\section{Does Eating Vegetables at Start of Meal Prevent Childhood Overweight in Japan? A-CHILD Study}

\author{
Yukako Tani ${ }^{1,2}$, Takeo Fujiwara ${ }^{1 *}$, Manami Ochi ${ }^{3}$, Aya Isumi ${ }^{1}$ and Tsuguhiko Kato ${ }^{4}$ \\ ${ }^{1}$ Department of Global Health Promotion, Tokyo Medical and Dental University (TMDU), Tokyo, Japan, ${ }^{2}$ Japan Society for the \\ Promotion of Science, Tokyo, Japan, ${ }^{3}$ Japan Support Center for Suicide Countermeasures, National Institute of Mental \\ Health, National Center of Neurology and Psychiatry, Tokyo, Japan, ${ }^{4}$ Department of Social Medicine, National Center for \\ Child Health and Development, Tokyo, Japan
}

Background: Because eating behaviors are established early in life, it is important to instill healthy eating habits in children. However, no published studies have examined the effects of what is habitually consumed first at a meal on children's body weight in real settings. The aim of this study was to examine the associations between what was consumed (vegetables, rice/bread, meat/fish, or soup) at the start of a meal and childhood overweight in Japan.

Methods: We used cross-sectional data from the Adachi Child Health Impact of Living Difficulty (A-CHILD) study, a population-based study comprising all first-grade students in Adachi City, Tokyo, Japan, performed in 2015. Through a questionnaire, we identified what types of food children ate first at meals. The questionnaire was completed by 4,040 caregivers. We used corresponding school health check-up data (height and weight) to assess overweight in each child.

Results: The proportions of what was consumed first at a meal were 11.6, 23.3, 25.4, 9.8, and 29.9\% for vegetables, meat/fish, rice/bread, soup, and undetermined (variable), respectively. Multivariate logistic regression showed the odds ratio of being overweight was 1.83 in children who ate meat/fish first (95\% Cl: 1.27-2.64, $p<0.01)$ compared with children who ate vegetables first. In contrast, the odds ratios in children who consumed rice/bread or soup first compared with children who ate vegetables first were 1.11 (95\% Cl: $0.76-1.61, p=0.59)$ and 1.29 (95\% Cl: 0.83-2.01, $p=0.26)$, respectively.

Conclusion: Children who eat meat/fish at the start of a meal are more likely to be overweight than those who eat vegetables at the start of a meal. Future studies are needed to investigate the mechanisms of how the order in which food is consumed at a meal affects weight status in children.

Keywords: eating behavior, order of food consumed, food sequencing, behavioral economics, childhood obesity

\section{INTRODUCTION}

The prevalence of childhood overweight and obesity has increased worldwide in recent decades and it has become a major public health epidemic (1-3). Between 1980 and 2013, the prevalence of overweight and obesity in developed countries has increased from 16.9 to $23.8 \%$ in boys and from 16.2 to $22.6 \%$ in girls (3). Overweight and obese children are likely to stay obese into adulthood 
(4-6), and have greater risk of heart disease, type 2 diabetes, stroke, and premature death and disability in adulthood $(7,8)$. Furthermore, children nowadays are more likely to become overweight at a younger age (9). Therefore, prevention of overweight in young children is important.

Although several risk factors for childhood overweight have been identified (10), the effectiveness of intervention programs has been minor and short-term (11). There is a need to establish a feasible and effective strategy against childhood overweight using an alternative approach. A notable example is the "CAN Approach," which is based on the practice of making healthy foods more convenient, attractive, and normative. This method nudges healthy food choices through behavioral economic theories of decision-making (12). For example, placing the healthiest food first and the least healthy food last at a buffet spread nudged people to select healthier meals, due to a high percentage of people taking the first available food (13). Similarly, the order of food consumption at meals can be habitual in conjunction with mealtime socialization (14), and this seems to be a normative choice for children. Therefore, we hypothesized that eating vegetables first at meals may increase vegetable intake, which aids in the prevention of overweight.

The order of food consumption may have an effect on overweight by changing the amount of food consumed during the rest of the meal. Some controlled studies in adults have shown that eating low energy density food first (vegetables, soup, or fruit) reduces total meal energy intake (15-17). Furthermore, Roe et al. reported that consuming low energy density salad before, rather than with, the main course increases total vegetable consumption (18).

The order of food consumption may also affect overweight and obesity by changing postprandial digestibility and metabolic effects. Glycemic excursions and incremental glucose peaks are reportedly significantly smaller when vegetables are consumed before carbohydrate foods compared with the reverse sequence (19). This may be due to the fiber in vegetables, which affects the speed of digestion and the amount of insulin needed for subsequent metabolic disposal (20).

Eating patterns established in early life tend to persist into adolescence and adulthood (21-23). Therefore, it is important to instill healthy eating habits in children. To date, however, no published studies have examined the effects of order of food consumption at meals on children's body weight in real settings.

In Japan, a typical meal-known as "ichi-ju san-sai" — consists of a staple food (such as rice), a soup (usually miso), and three dishes (one main side dish and two side dishes). The food is served all at once (24). Therefore, the order of food consumption at a meal can vary significantly among individuals.

To that end, a health promotion strategy known as "Eat Vegetables First at Meals" was launched in Adachi City in Tokyo, Japan, in 2013 to address various health problems. Specifically, the municipality focused on children in public daycare centers. To assess the impact of this health promotion policy, a questionnaire survey concerning eating habits of children in Grade 1 in Adachi City was implemented. The responses to the questionnaire was linked with school health checks data, including objective assessments of height and weight.
The purpose of the present study was to use this populationbased sample to examine the associations between the order of food consumption at a meal in Grade 1 elementary school children and their overweight status in Japan.

\section{MATERIALS AND METHODS}

\section{Study Design and Subjects}

We used data from the Adachi Child Health Impact of Living Difficulty (A-CHILD) study performed in Adachi City, Tokyo, Japan, in 2015 (25). The survey covered all 69 public elementary schools in Adachi City. Between July 2015 and November 2015, self-reported questionnaires with anonymous unique ID were distributed to 5,355 children in the first grade of elementary school (aged 6 to 7 years). Children were asked to pass on the questionnaires to their caregivers at home to fill out. The children then returned the completed questionnaires to their school. A total of 4,467 questionnaires were returned (response rate: $83.4 \%$ ) and 4,291 of the responding caregivers provided informed consent to include their child in the study (25). Questionnaire responses were linked to each participating child's school health check-up data from the same year (linkage rate: 100\%) to determine their body mass indexes (BMI). The analyses were carried out using the data of 4,040 participants after excluding the following: (i) potential participants whose sex, date of birth, or body weight status were missing ( $n=189$ ); and (ii) potential participants whose caregivers had not answered the questions about what was consumed first $(n=62)$. The A-CHILD protocol was approved by the Ethics Committee of the National Center for Child Health and Development (No. 1187). All participants gave written informed consent in accordance with the Declaration of Helsinki.

\section{Child Body Weight Status}

School teachers assessed elementary school children's height and weight during health check-ups according to a standardized protocol (26). Height was measured to the nearest $0.1 \mathrm{~cm}$ using a portable stadiometer, and weight to the nearest $0.1 \mathrm{~kg}$ on a digital scale without shoes and wearing light clothing. BMI was calculated from weight (in kilograms) over square of height (in meters). BMI was expressed as z-score representing the deviation in standard deviation units from the mean of a standard normal distribution of BMI specific to age and sex according to the standard of the WHO Child Growth Standards. Children were categorized as non-overweight or overweight using +1 standard deviation cut-off points (27).

\section{Order of Food Consumption}

What was consumed first at meals over the past month was assessed using the following question: "What does your child eat first at mealtimes? Circle the answer that best applies for the past month." The responses to this question were "vegetables," "meat/fish," "rice/bread (staple)," "soup," or "not determined (variable)." 


\section{Covariates}

Because the "Eat Vegetables First at Meals" campaign was launched prior to our study, adjustments were made for the preschool status (public nursery, private nursery, or private kindergarten) of each child. Potential confounders of the association between what is consumed first at meals and overweight were assessed by questionnaires completed by caregivers.

The children's physical activity was assessed according to the frequency of being physically active for half an hour or more, and was categorized into four groups (never/rarely, 1-2, 3-4, or $\geq 5$ times/week). The status of the responder to the questionnaire was categorized into two groups (mother or father).

Marital status of caregivers was categorized into two groups (married/common-law marriage or unmarried/divorced/bereavement). Annual normalized household income was determined from the household income and the number of household members and divided into three categories ( $<2.50,2.50-3.49$, or $\geq 3.50$ million yen).

Family meal status during dinner (children eating with family or only with other children/alone) was included in the analysis because family meals are related to children's eating patterns and body weight status (28). Because children are largely cared for by their mothers and only $10 \%$ of fathers take care of children's meals in Japan (29), mother's characteristics were included: age $(<30,30-39$, or $\geq 40$ years), educational status (low: junior high school/high school; middle: technical/junior college/college dropout; or high: college/graduate school), and employment status (full-time, part-time, self-employed, side job, or unemployed). Participating children's BMI at age 3 years was calculated using the height and weight reported by their caregivers. BMI was expressed as z-score representing the deviation in standard deviation units from the mean of a standard normal distribution of BMI specific to age and sex according to the standard of the WHO Child Growth Standards. Children were categorized as non-overweight or overweight using +1 standard deviation cut points (30). Mothers' and fathers' BMI were calculated using their self-reported heights and weights. Standard categories of BMI (31) were used to characterize parents as obese $\left(B M I \geq 30.0 \mathrm{~kg} / \mathrm{m}^{2}\right)$, overweight $(\mathrm{BMI}=25.0-29.9$ $\left.\mathrm{kg} / \mathrm{m}^{2}\right)$, normal $\left(\mathrm{BMI}=18.5-24.9 \mathrm{~kg} / \mathrm{m}^{2}\right)$, and underweight $\left(\mathrm{BMI}<18.5 \mathrm{~kg} / \mathrm{m}^{2}\right)$.

\section{Statistical Analysis}

First, $\chi^{2}$-tests were used to assess participants' characteristics and differences in what is consumed first at meals. Second, the association between what is consumed first at meals and the overweight status of each child was evaluated using logistic regression analysis to calculate the adjusted odds ratios (OR) with $95 \%$ confidence intervals (CI). The following sequence of models was constructed: Model 1 was adjusted for the sex of each child; Model 2 was additionally adjusted for each child's physical activity and preschool status, each respondent's (parent's) status and marital status, annual normalized household income, family meal, mother's age, mother's education and mother's employment; and Model 3 was further adjusted for each child's BMI at 3 years, and mother's and father's BMI. All analyses were conducted using STATA version 13 (Stata Statistical Software: Release 13. College Station, TX: StataCorp LP).

\section{RESULTS}

Overall, the proportions of what was consumed first at meals were $11.6,23.3,25.4,9.8$, and $29.9 \%$ for vegetables, meat/fish, rice/bread, soup, and not determined, respectively (Table 1). Of the participating children, 13.5\% were overweight. Most respondents were mothers $(90.7 \%)$. The proportions of preschool status were $14.0 \%, 25.9 \%$, and $55.8 \%$ for private nursery, public nursery, and private kindergarten, respectively. Of the children who ate vegetables first at meals, 30.2\% previously attended public nurseries, whereas $22.9 \%$ of children who ate meat/fish first at meals previously attended public nurseries.

After adjusting for each child's sex, children who ate meat/fish first at meals were 1.82 times (95\% CI: $1.30-2.54, p<0.001$ ) more likely to be overweight than those who ate vegetables first at meals (Model 1, Table 2). In contrast, the OR in children who ate rice/bread or soup first at first compared with children who ate vegetables first at meals were 1.18 (95\% CI: $0.84-1.67, p=0.34$ ) and 1.30 (95\% CI: 0.86-1.95, $p=0.21$ ), respectively (Model 1 , Table 2). Adjusting for each child's physical activity, preschool status, each respondent's (parent's) status and marital status, annual normalized household income, mother's age, mother's education, mother's employment and family meal, the OR in children who ate meat/fish first was slightly lower, but remained significant (1.80; 95\% CI: 1.28-2.54, $p<0.01$ ) (Model 2). Subsequent adjustment for each child's BMI at age 3 years and mother's and father's BMI did not weaken the association (1.83; 95\% CI: 1.27-2.64, $p<0.01$ ) (Model 3).

\section{DISCUSSION}

In this study, we found that children who habitually eat meat/fish first at meals are more likely to be overweight than those who eat vegetables first. In contrast, the effects of eating rice/bread or soup first on overweight did not differ significantly from that of eating vegetables first. To our knowledge, this is the first study to examine the effect of what is consumed first at meals on body weight status in children in a real setting.

There are several possible explanations for our findings. One possibility is that eating low energy density food (vegetables) first at meals may better promote satiety and reduce energy intake over the course of the meal compared with eating high energy density food (meat/fish) first. Indeed, a suggested strategy for enhancing satiety and reducing energy intake is to consume a filling portion of low energy density food, such as vegetables or salad, as a first course (32). Furthermore, in a single-meal study, serving children a low energy density, vegetable-based soup as a first course was associated with less energy intake from the more energy dense main course (33). In adults, consuming low energy density salads is associated with less energy intake over a meal than having no first course (15). 


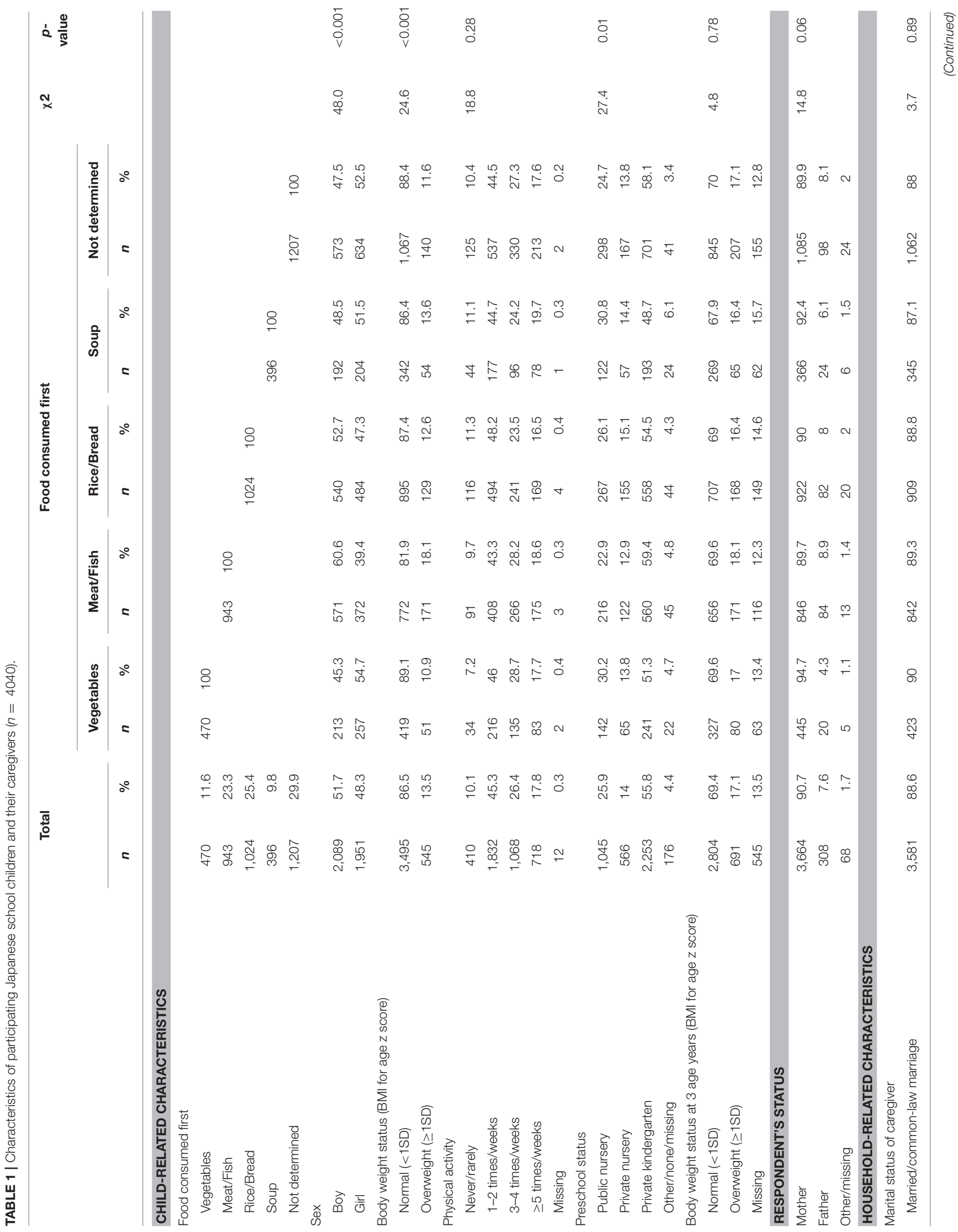




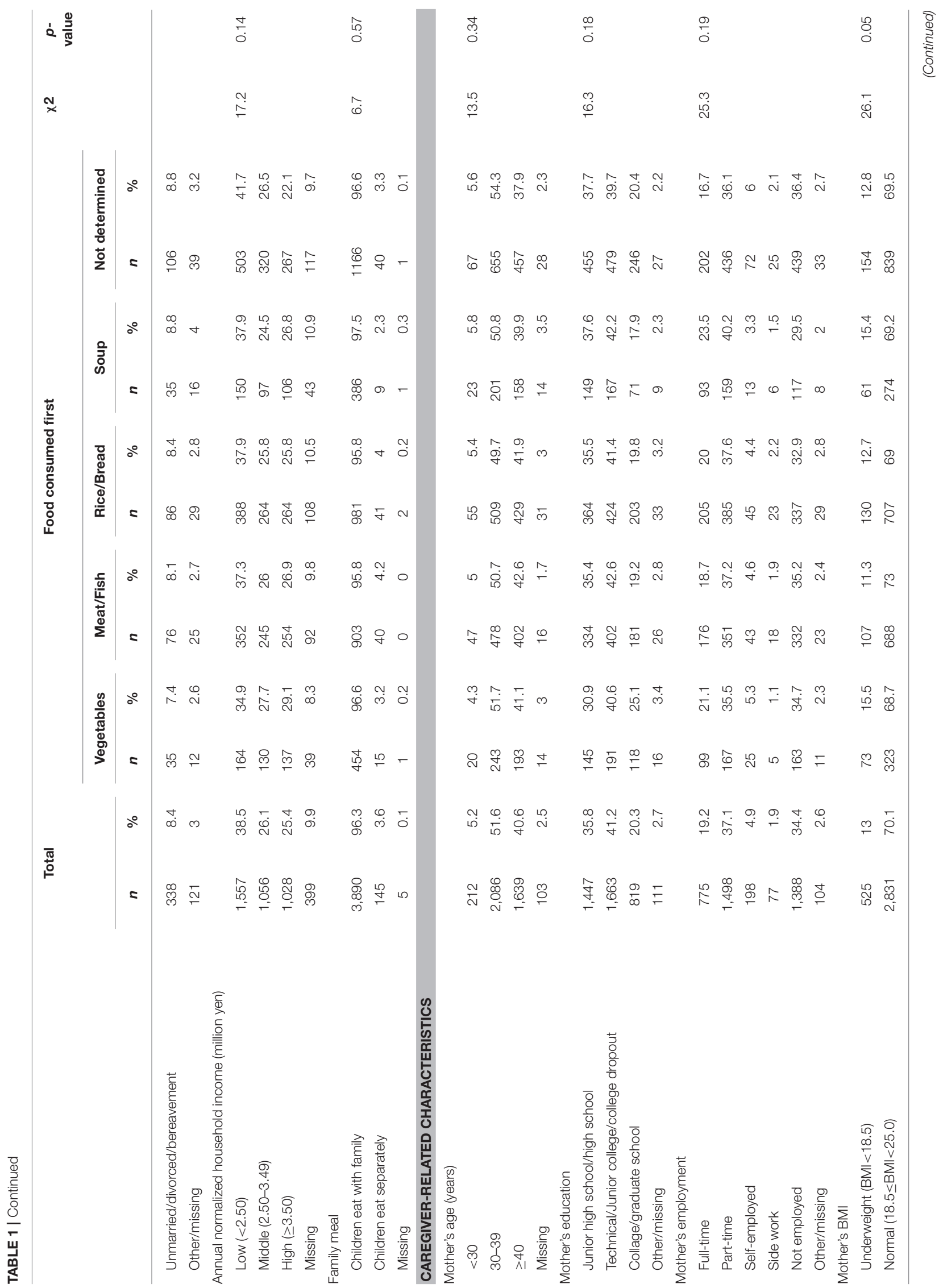




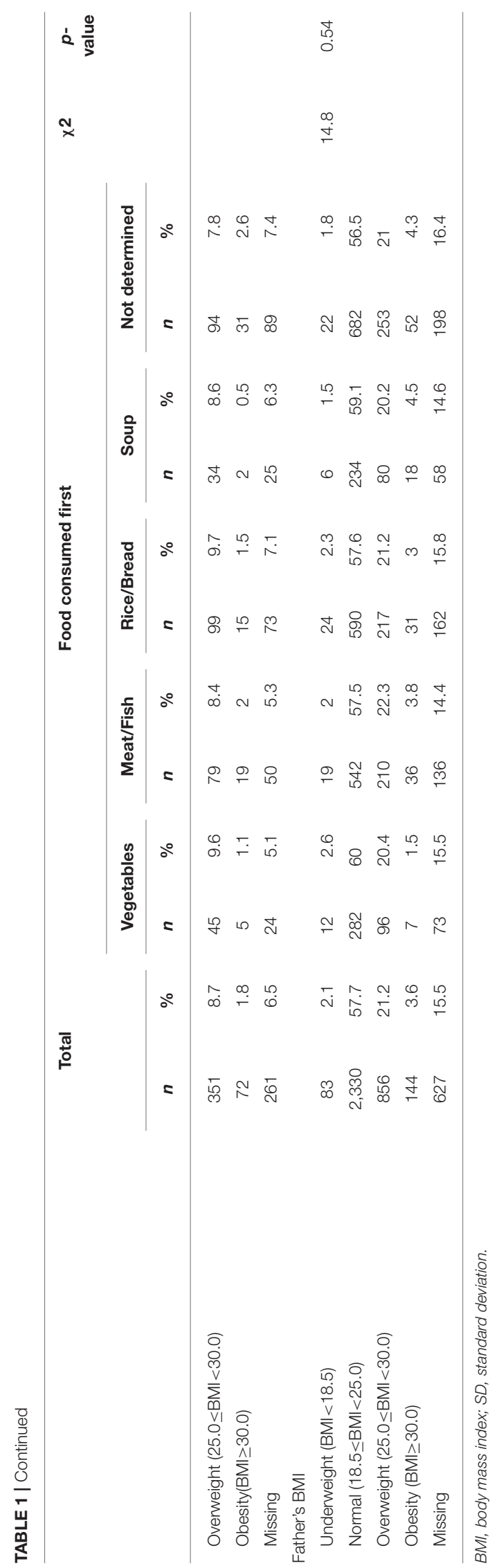

These findings are consistent with our present study, which found that the effect on overweight of eating soup, a low energydensity food, first did not differ significantly from that of eating vegetables first. In addition to vegetables, eating other low energy density foods (such as soup or fruit) as a first course is also associated with eating less of a higher energy density main course and less energy intake over the course of the meal $(16,17)$. In several multiple-meal studies of both adults and children, reducing the energy density of multiple meals over 2 days resulted in decreased cumulative energy intake $(34,35)$, suggesting that reducing the energy density of multiple meals leads to persistent and habitual decreases in energy intake from meals. This may in turn contribute to the prevention of overweight. Future studies are needed to investigate energy intake over a meal to clarify the mechanisms of the effect of food sequencing on overweight.

Another possibility is that eating vegetables first provides greater opportunity to eat more vegetables. In a crossover design, serving vegetables or vegetable-based-soup as a first course was associated with increased total vegetable consumption over the meal by children $(33,36)$. Children typically like fatty and sugary foods and dislike vegetables (37) because they innately prefer sweet and salty foods and reject sour and bitter foods (38). One Japanese study showed that vegetables were the most disliked of all foods in school lunches and that dislike was the main reason for leftovers among primary school children (39). Therefore, children who eat meat/fish first may leave more vegetables than those who eat vegetables first.

Furthermore, our measure of what was consumed first at meals may act as a proxy for the habitual quality of family meals. For example, the frequency of vegetables being served as part of family meals may be higher among children who eat vegetables first, and may in fact result in these children acquiring the habit of eating vegetables first. We found the prevalence of eating vegetables at both breakfast and dinner (over a month) was higher in children who ate vegetables first than in those who ate meat/fish first ( 48 vs. $31 \%$, respectively; $p<0.001$ ). Additionally, the consumption of obesity-related foods (juice, dessert, etc.) may be higher in children who eat meat/fish first. Further studies on the habitual quality of meals are needed to explore this possibility.

Eating rice/bread first at meals did not differ significantly from eating vegetables first in terms of the impact on overweight. Because eating vegetables before carbohydrates is associated with smaller glycemic excursions and glucose peaks than the reverse sequence (19), we hypothesized that eating vegetables first at meals would better protect against overweight than eating carbohydrates (rice/bread) first. However, the effects of eating vegetables first may not have depended on digestibility and metabolic effects in the present setting. The mechanisms underlying the differences in effects of what is consumed first still require further investigation.

This study has several limitations. First, we did not explore the validity or reliability of the question assessing what is consumed first at meals. We collected information on what 
TABLE 2 | Adjusted odds ratios for overweight status according to the food consumed first by Japanese school children $(n=4040)$.

\begin{tabular}{|c|c|c|c|c|c|c|}
\hline & \multicolumn{2}{|c|}{ Model 1} & \multicolumn{2}{|c|}{ Model 2} & \multicolumn{2}{|c|}{ Model 3} \\
\hline & \multicolumn{2}{|c|}{ Overweight } & \multicolumn{2}{|c|}{ Overweight } & \multicolumn{2}{|c|}{ Overweight } \\
\hline & OR $(95 \% \mathrm{Cl})$ & $p$-value & OR $(95 \% \mathrm{Cl})$ & $p$-value & OR $(95 \% \mathrm{Cl})$ & $p$-value \\
\hline \multicolumn{7}{|c|}{ FOOD CONSUMED FIRST } \\
\hline Vegetables & Reference & & Reference & & Reference & \\
\hline Meat/Fish & $1.82(1.30-2.54)$ & $<0.001$ & $1.80(1.28-2.54)$ & $<0.01$ & $1.83(1.27-2.64)$ & $<0.01$ \\
\hline Rice/Bread & $1.18(0.84-1.67)$ & 0.34 & $1.14(0.80-1.61)$ & 0.46 & $1.11(0.76-1.61)$ & 0.59 \\
\hline Soup & $1.30(0.86-1.95)$ & 0.21 & $1.23(0.81-1.85)$ & 0.33 & $1.29(0.83-2.01)$ & 0.26 \\
\hline Not determined & $1.08(0.77-1.51)$ & 0.67 & $1.06(0.75-1.50)$ & 0.74 & $0.99(0.68-1.43)$ & 0.95 \\
\hline
\end{tabular}

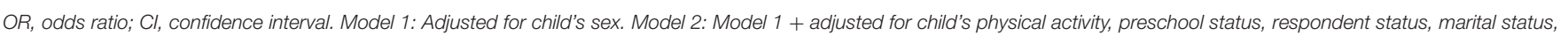

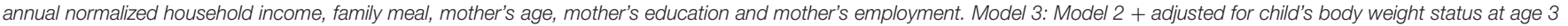
years, mother's and father's BMI.

children ate first from their caregivers. Therefore, the responses may have been biased due to the caregivers' perception. Social desirability bias may also have skewed the caregivers' responses. Nonetheless, we confirmed what was consumed first did not differ according to the caregiver's social status (marital status, income, mother's age, education, and employment), and the prevalence of eating vegetables first was higher in children from public nurseries, where a habituation policy of eating vegetables first had been implemented. Second, we lacked information on the amount of food that was consumed first. Therefore, we included children who ate both large and small amounts of vegetables first in the same category. For example, the "vegetables first" category may include children who had a bite of vegetables at the beginning of meals but ate the rest of the vegetables later. However, our hypothesis is not about the effectiveness of the amount of vegetables consumed first, but rather the habit of eating vegetables first, which leads to a higher frequency of eating vegetables. Future studies should aim to elucidate the mechanism by which habitually eating vegetables first increases the frequency or amount of vegetables consumed, which eventually prevents overweight among children. Third, because we lacked information on factors such as children's food preferences and caregivers' knowledge about nutrition, we may not have taken all confounding factors into account. For example, caregivers who know a lot about nutrition may not only pay attention to what their children eat, but may also teach them to eat vegetables first. Fourth, despite the difference in the nutritional value of fish and meat (chicken, red meat, etc.), we grouped these food categories together. Future study should divide these categories to clarify the mechanism of the effect of eating meat and fish first on overweight. Finally, we did not assess energy intake or physical exercise, and the study design was crosssectional. Hence, the mechanism by which the habit of eating vegetables first prevents overweight among children is yet to be uncovered.

In conclusion, what was consumed first at meals was associated with children's body weight status. Based on the theories of behavioral economics, the habitual behavior of avoiding meat/fish first may nudge children to eat better. This strategy may be useful for "non-course-meal" culture in which all dishes are served at one time, such as the meals typically served in Japan. Further studies measuring total daily and mealtime energy intake, energy per food category, energy expenditure, food preferences, glucose metabolism, and satiety responsiveness based on food group order are warranted to determine whether and how eating vegetables first actually prevents childhood overweight.

\section{AUTHOR CONTRIBUTIONS}

YT and TF conceived the design. TF, MO, AI, and TK collected data. YT reviewed literature and analyzed data. YT wrote first draft of paper. TF revised the first draft. MO, AI, and TK edited the manuscript. All authors approved the final version of the manuscript.

\section{FUNDING}

This study was supported by a Health Labour Sciences Research Grant, Comprehensive Research on Lifestyle Disease from the Japanese Ministry of Health, Labour and Welfare (H27-Jyunkankito-ippan-002), and Grants-in-Aid for Scientific Research from the Japan Society for the Promotion of Science (JSPS KAKENHI Grant Number 16H03276, 16K16295, and 16J11423), St. Luke's Life Science Institute Grants, and the Japan Health Foundation Grants.

\section{ACKNOWLEDGMENTS}

We are particularly grateful to the staff members and central office of Adachi City Hall for conducting the survey. We would like to thank everyone who participated in the surveys. In particular, we would also like to thank Mayor Yayoi Kondo, Mr. Syuichiro Akiu, Mr. Hideaki Otaka, and Ms. Yuko Baba of Adachi City Hall, all of whom contributed significantly to completion of this study. 


\section{REFERENCES}

1. de Onis M, Blossner M, Borghi E. Global prevalence and trends of overweight and obesity among preschool children. Am J Clin Nutr. (2010) 92:1257-64. doi: 10.3945/ajcn.2010.29786

2. Wang Y, Lobstein T. Worldwide trends in childhood overweight and obesity. Int J Pediatr Obes. (2006) 1:11-25. doi: 10.1080/17477160600586747

3. Ng M, Fleming $\mathrm{T}$, Robinson $\mathrm{M}$, Thomson B, Graetz N, Margono $\mathrm{C}$, et al. Global, regional, and national prevalence of overweight and obesity in children and adults during 1980-2013: a systematic analysis for the Global Burden of Disease Study 2013. Lancet (2014) 384:766-81. doi: 10.1016/s0140-6736(14)60460-8

4. Serdula MK, Ivery D, Coates RJ, Freedman DS, Williamson DF, Byers T. Do obese children become obese adults? A review of the literature. Prev Med. (1993) 22:167-77. doi: 10.1006/pmed.1993.1014

5. Herman KM, Craig CL, Gauvin L, Katzmarzyk PT. Tracking of obesity and physical activity from childhood to adulthood: the Physical Activity Longitudinal Study. Int J Pediatr Obes. (2009) 4:281-8. doi: 10.3109/17477160802596171

6. Mossberg HO. 40-year follow-up of overweight children. Lancet (1989) 2:491-3.

7. Dietz WH. Health consequences of obesity in youth: childhood predictors of adult disease. Pediatrics (1998) 101:518-25.

8. Pandita A, Sharma D, Pandita D, Pawar S, Tariq M, Kaul A. Childhood obesity: prevention is better than cure. Diabetes Metab Syndr Obes. (2016) 9:83-9. doi: $10.2147 / \mathrm{dmso} . \mathrm{s} 90783$

9. Kim J, Must A, Fitzmaurice GM, Gillman MW, Chomitz V, Kramer E, et al. Incidence and remission rates of overweight among children aged 5 to 13 years in a district-wide school surveillance system. Am J Public Health (2005) 95:1588-94. doi: 10.2105/ajph.2004.054015

10. Ebbeling CB, Pawlak DB, Ludwig DS. Childhood obesity: publichealth crisis, common sense cure. Lancet (2002) 360:473-82. doi: 10.1016/s0140-6736(02)09678-2

11. Mead E, Brown T, Rees K, Azevedo LB, Whittaker V, Jones D, et al. Diet, physical activity and behavioural interventions for the treatment of overweight or obese children from the age of 6 to 11 years. Cochrane Database Syst Rev (2017) 6:Cd012651. doi: 10.1002/14651858.cd012651

12. Wansink B. Convenient, attractive, and normative: the CAN approach to making children slim by design. Child Obes. (2013) 9:277-8. doi: 10.1089/chi.2013.9405

13. Wansink B, Hanks AS. Slim by design: serving healthy foods first in buffet lines improves overall meal selection. PLoS ONE (2013) 8:e77055. doi: 10.1371/journal.pone.0077055

14. Ochs E, Shohet M. The cultural structuring of mealtime socialization. New Dir Child Adolesc Dev. (2006) 2006:35-49. doi: 10.1002/cd.154

15. Rolls BJ, Roe LS, Meengs JS. Salad and satiety: energy density and portion size of a first-course salad affect energy intake at lunch. J Am Diet Assoc. (2004) 104:1570-6. doi: 10.1016/j.jada.2004.07.001

16. Flood JE, Rolls BJ. Soup preloads in a variety of forms reduce meal energy intake. Appetite (2007) 49:626-34. doi: 10.1016/j.appet.2007.04.002

17. Flood-Obbagy JE, Rolls BJ. The effect of fruit in different forms on energy intake and satiety at a meal. Appetite (2009) 52:416-22. doi: 10.1016/j.appet.2008.12.001

18. Roe LS, Meengs JS, Rolls BJ. Salad and satiety. The effect of timing of salad consumption on meal energy intake. Appetite (2012) 58:242-8. doi: 10.1016/j.appet.2011.10.003

19. Imai S, Fukui M, Kajiyama S. Effect of eating vegetables before carbohydrates on glucose excursions in patients with type 2 diabetes. J Clin Biochem Nutr. (2014) 54:7-11. doi: 10.3164/jcbn.13-67

20. Wong JM, Jenkins DJ. Carbohydrate digestibility and metabolic effects. J Nutr. (2007) 137:2539s-46s. doi: 10.1093/jn/137.11.2539S

21. Mikkila V, Rasanen L, Raitakari OT, Pietinen P, Viikari J. Consistent dietary patterns identified from childhood to adulthood: the cardiovascular risk in Young Finns Study. Br J Nutr. (2005) 93:923-31. doi: 10.1079/BJN20051418
22. Wang Y, Bentley ME, Zhai F, Popkin BM. Tracking of dietary intake patterns of Chinese from childhood to adolescence over a six-year follow-up period. $J$ Nutr. (2002) 132:430-8. doi: 10.1093/jn/132.3.430

23. Lien N, Lytle LA, Klepp KI. Stability in consumption of fruit, vegetables, and sugary foods in a cohort from age 14 to age 21. Prev Med. (2001) 33:217-26. doi: 10.1006/pmed.2001.0874

24. Ministry of Agriculture, Forestry and Fisheries. WASHOKU. Available online at: http://www.maff.go.jp/j/keikaku/syokubunka/culture/pdf/guide_ all.pdf (Accessed February 6, 2018). (in Japanese).

25. Doi S, Fujiwara T, Ochi M, Isumia A, Katoc T. Association of sleep habits with behavior problems and resilience of 6- to 7-year-old children: Results from the A-CHILD study. Sleep Med. (2018) 45:62-8. doi: 10.1016/j.sleep.2017.12.015

26. Education and Science in Ministry of Sports and Youth Bureau of School Health Education. Children's Health Diagnostic Manual (Revised edition). Tokyo: Japanese Society of School Health (2006).

27. de Onis M, Onyango AW, Borghi E, Siyam A, Nishida C, Siekmann J. Development of a WHO growth reference for school-aged children and adolescents. Bull World Health Organ. (2007) 85:660-7.

28. Hammons AJ, Fiese BH. Is frequency of shared family meals related to the nutritional health of children and adolescents? Pediatrics (2011) 127:e156574. doi: $10.1542 /$ peds.2010-1440

29. National Women's Education Center. International Comparative Study on Child Raising and Family Life 2005. Tokyo, Japan, (2006).

30. WHO Multicentre Growth Reference Study Group. WHO Child Growth Standards: Head Circumference-for-Age, Arm Circumference-for-Age, Triceps Skinfold-for-Age and Subscapular Skinfold-for-Age: Methods and Development. Geneva: World Health Organization (2007).

31. WHO. Obesity: Preventing and Managing the Global Epidemic. Geneva: World Health Organization (2000).

32. Rolls BJ, Bell EA, Thorwart ML. Water incorporated into a food but not served with a food decreases energy intake in lean women. Am J Clin Nutr. (1999) 70:448-55. doi: 10.1093/ajcn/70.4.448

33. Spill MK, Birch LL, Roe LS, Rolls BJ. Serving large portions of vegetable soup at the start of a meal affected children's energy and vegetable intake. Appetite (2011) 57:213-9. doi: 10.1016/j.appet.2011.04.024

34. Rolls BJ, Roe LS, Meengs JS. Reductions in portion size and energy density of foods are additive and lead to sustained decreases in energy intake. Am J Clin Nutr. (2006) 83:11-7. doi: 10.1093/ajcn/83.1.11

35. Leahy KE, Birch LL, Rolls BJ. Reducing the energy density of multiple meals decreases the energy intake of preschool-age children. Am J Clin Nutr. (2008) 88:1459-68. doi: 10.3945/ajcn.2008.26522

36. Spill MK, Birch LL, Roe LS, Rolls BJ. Eating vegetables first: the use of portion size to increase vegetable intake in preschool children. Am J Clin Nutr (2010) 91:1237-43. doi: 10.3945/ajcn.2009.29139

37. Cooke LJ, Wardle J. Age and gender differences in children's food preferences. Br J Nutr (2005) 93:741-6. doi: 10.1079/BJN200 51389

38. Birch LL. Development of food preferences. Annu Rev Nutr. (1999) 19:41-62. doi: 10.1146/annurev.nutr.19.1.41

39. Japan Sport Council. The 2010 Child Dietary Survey in Japan. (2010) Available online at: http://www.jpnsport.go.jp/anzen/school_lunch//tabid/ 1490/Default.aspx

Conflict of Interest Statement: The authors declare that the research was conducted in the absence of any commercial or financial relationships that could be construed as a potential conflict of interest.

Copyright (c) 2018 Tani, Fujiwara, Ochi, Isumi and Kato. This is an open-access article distributed under the terms of the Creative Commons Attribution License (CC $B Y)$. The use, distribution or reproduction in other forums is permitted, provided the original author(s) and the copyright owner are credited and that the original publication in this journal is cited, in accordance with accepted academic practice. No use, distribution or reproduction is permitted which does not comply with these terms. 